\title{
Nonionizing photoacoustic cystography in vivo
}

\author{
Chulhong Kim, ${ }^{1, *}$ Mansik Jeon, ${ }^{1}$ and Lihong V. Wang ${ }^{2}$ \\ ${ }^{1}$ BioOptics and Acoustics Laboratory, Department of Biomedical Engineering, University at Buffalo, \\ The State University of New York, Buffalo, New York 14260, USA \\ ${ }^{2}$ Optical Imaging Laboratory, Department of Biomedical Engineering, Washington University in St. Louis, \\ St. Louis, Missouri 63130, USA \\ ${ }^{*}$ Corresponding author: chulhong@buffalo.edu
}

Received June 24, 2011; revised August 7, 2011; accepted August 16, 2011; posted August 17, 2011 (Doc. ID 149910); published September 12, 2011

We demonstrate the feasibility of a novel and nonionizing process for bladder imaging in vivo, called photoacoustic cystography (PAC). Using a photoacoustic imaging system, we have successfully imaged a rat bladder filled with clinically used Methylene Blue (MB) dye. An image contrast of $\sim 8$ was achieved. Further, spectroscopic PAC confirmed the accumulation of $\mathrm{MB}$ in the bladder. Using a laser pulse energy of less than $1 \mathrm{~mJ} / \mathrm{cm}^{2}(1 / 20$ of the ANSI safety limit), a deeply $(1.2 \mathrm{~cm})$ positioned bladder in biological tissues was clearly visible in the PA image. Our results suggest that PAC can potentially provide a nonionizing, relatively cheap, and portable tool for bladder mapping. Among our clinical interests, nonionizing PAC with an injection of MB can potentially monitor vesicoureteral reflux in children. (c) 2011 Optical Society of America

OCIS codes: $110.0110,170.0170,170.5120$.

Cystoscopy is a gold standard for bladder imaging, yet this method provides magnified surface properties only. $\mathrm{X}$-ray computed tomography has been applied, but it is ionizing and not sensitive. Contrast-enhanced MRI has been applied, but it is expensive and bulky [1]. Although ultrasound (US) imaging is real-time, portable, relatively cost effective, and safe, conventional US imaging is not sensitive, especially, for imaging bladder cancers [2].

Photoacoustic tomography (PAT) is a hybrid nonionizing imaging modality that combines the merits of both light and US, and provides an approach for mapping the optical absorption contrast [3]. In PAT, a short pulsed laser source is used to illuminate the tissue sample, and the photoacoustic (PA) waves excited by thermoelastic expansion due to light absorption are then measured to reconstruct the optical absorption distribution. Because ultrasonic waves are much less scattered than light in biological tissues, the relative spatial resolution (the ratio between depth and axial resolution) is maintained approximately at 200 , while the penetration depth of PAT is extended to several centimeters by using diffuse rather than ballistic light. Recently, PAT has been extensively explored in preclinical and clinical studies, including primary cancer detection, lymph node staging and lymphangiography, brain activation, ophthalmology, and image-guided therapy [4-8]. A clinical ultrasonic scanner has been adapted to PAT, combining the high contrast of optical imaging and the high resolution of US imaging [9-11].

In this Letter, for the first time to our knowledge, we describe the feasibility of a novel and nonionzing approach for bladder imaging using PAT, referred to as photoacoustic cystography (PAC). A healthy rat bladder filled with Methylene Blue (MB) was photoacoustically and spectroscopically imaged in vivo without any ionizing radiation. The imaging depth was also extended beyond $1 \mathrm{~cm}$ with a laser pulse energy of $<1 \mathrm{~mJ} / \mathrm{cm}^{2}$. When combined with a clinical US scanner, this technique can potentially supply an accurate, safe, cheap, portable, and real-time imaging capabilities for bladder imaging.
A volumetric PAT system is shown in Fig. 1(a). The system setup is detailed in [12]. In brief, the light was delivered from a tunable optical parametric oscillator laser (Surelite OPO PLUS; Continuum; wavelength tunability: 410 to $2500 \mathrm{~nm}$ ) pumped by a $Q$-switched Nd:YAG laser (SLII-10; Continuum) with a 5 ns pulse duration and a $10 \mathrm{~Hz}$ pulse repetition rate. A light wavelength of $667 \mathrm{~nm}$ was used for most of the experiments, but both 667 and $850 \mathrm{~nm}$ wavelengths were used for spectroscopic bladder identification. Formed by a concave lens, a spherical conical lens, and an optical condenser, the darkfield light illumination was coaxially aligned with the US focus in water. The incident laser pulse energy was less than $1 \mathrm{~mJ} / \mathrm{cm}^{2}$, far below the ANSI safety limit of $20 \mathrm{~mJ} / \mathrm{cm}^{2}$ at this wavelength. Generated PA waves were detected by a single-element $5 \mathrm{MHz}$ US transducer (V308; Olympus NDT). The spatial resolutions were 144 and $590 \mu \mathrm{m}$ in the axial and transverse directions, respectively. The scanning speed for one PA image was $\sim 25 \mathrm{~min}$, with a FOV of $2.5 \mathrm{~cm} \times 2.5 \mathrm{~cm}$ in the $x-y$ plane. No signal was averaged.

Approval of the experimental procedure was obtained from an institutional animal care and use committee. Healthy Sprague Dawley rats that weighed $\sim 250$ g were used in all imaging experiments. First, the rats were given a mixture of ketamine $(85 \mathrm{mg} / \mathrm{kg}$ body weight $)$ and xylazine $(15 \mathrm{mg} / \mathrm{kg})$ for anesthesia. After depilation in the abdominal region, the rats were positioned atop a homemade holder [Fig. 1(b)]. The distal end of a 22-gauge catheter was coated with lubricant. After the urinary meatus was identified, the catheter was gently inserted until urine was noticed. Then, urine in the bladder was allowed to void through the catheter. Before injection, we obtained a control image. After injection of MB (clinically used for mapping sentinel lymph nodes in breast cancer patients [11]), with a concentration of $30 \mathrm{mM}$ and a dose of $0.2 \mathrm{ml}$ via the catheter, we acquired a series of PA images. We added chicken breast tissue on top of the rats to increase imaging depth. After all imaging experiments, the rat was sacrificed with an overdose of 

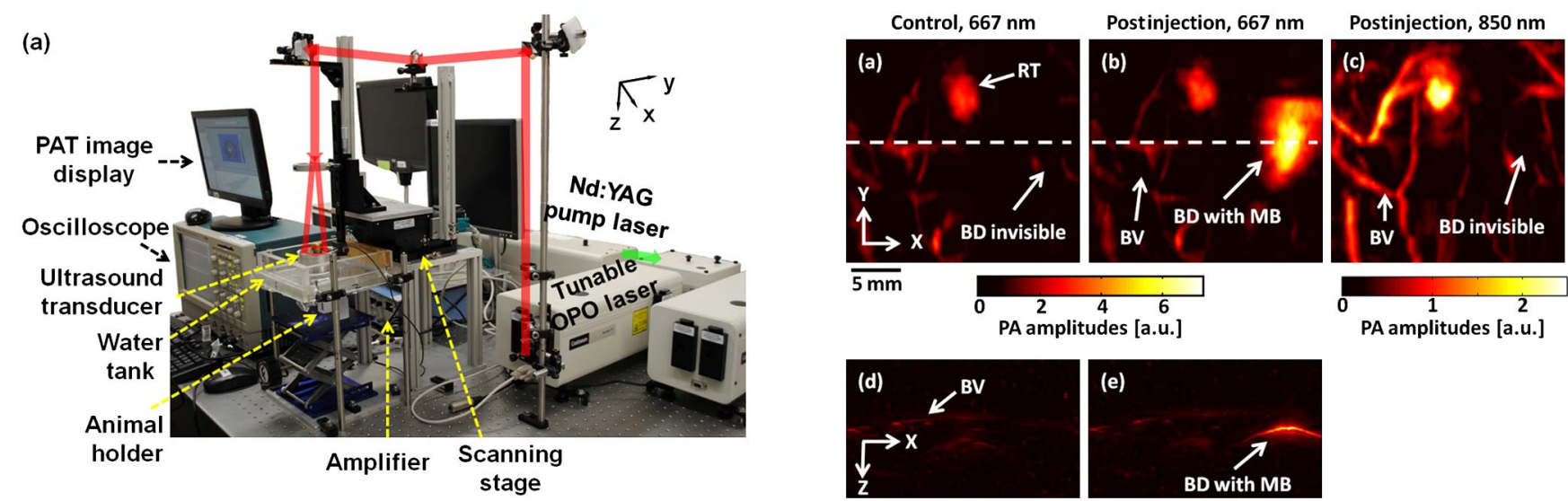

(b)

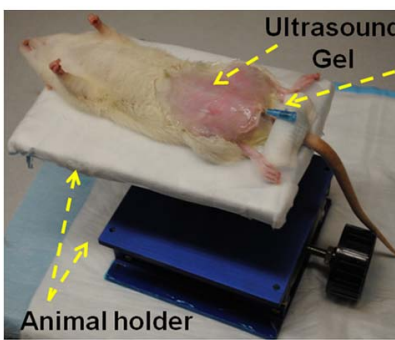

22-gauge catheter

Fig. 1. (Color online) (a) Photograph of the PAT system. (b) Photograph of an animal with bladder catheterization.

pentobarbital. Then, the skin was removed, and the bladders were visualized to confirm the occupation of MB.

The preinjection control PA image [Fig. 2(a)] was obtained at a wavelength of $667 \mathrm{~nm}$, where the optical absorption of MB is peaked, and is shown in the form of maximum amplitude projection (MAP), which projects the maximum signal along each $\mathrm{A}$ line onto the corresponding $x-y$ plane. Figure $2(\mathrm{f})$ is a photograph of a rat taken prior to PA image acquisition and after hair removal and catheterization. The vasculature in the abdominal area was clearly imaged with high contrast $(1.1 \pm 0.6$, standard deviation with respect to the background, based on acoustic amplitude). The bladder was invisible in the control PA image because urine in the bladder was optically transparent at the $667 \mathrm{~nm}$ wavelength. The bladder appeared unmistakably in the PA image acquired after MB injection. Because MB has higher optical absorption $\left(\sim 2191 \mathrm{~cm}^{-1}\right.$ at $\left.30 \mathrm{mM}\right)$ than blood $\left(\sim 3 \mathrm{~cm}^{-1}\right.$ for an artery with $90 \%$ hemoglobin oxygen saturation and $\sim 5.8 \mathrm{~cm}^{-1}$ for a vein with $70 \%$ hemoglobin oxygen saturation) at the $667 \mathrm{~nm}$ wavelength, the bladder appeared with a much higher contrast $(8.1 \pm 2.1)$ than the surrounding blood vessels [Fig. 2(b)]. Certainly, the PA signal from the bladder filled with MB was saturated. The contrast of the bladder with respect to the blood vessel was 3.4. After injection of MB, without a proper control image, the bladder was difficult to distinguish from the surrounding blood vasculature. However, the MB-filled bladder could be distinguished from surrounding blood vasculature by collecting images at two wavelengths $(667$ and $850 \mathrm{~nm})$. Because of the different optical absorption coefficients of MB at these wavelengths, the bladder could be effectively differentiated from blood vessels by comparing the two images. At $850 \mathrm{~nm}$, where the optical absorption of $\mathrm{MB}$ is much reduced, the PA signals from within the bladder disappeared [Fig. 2(c)]. This result
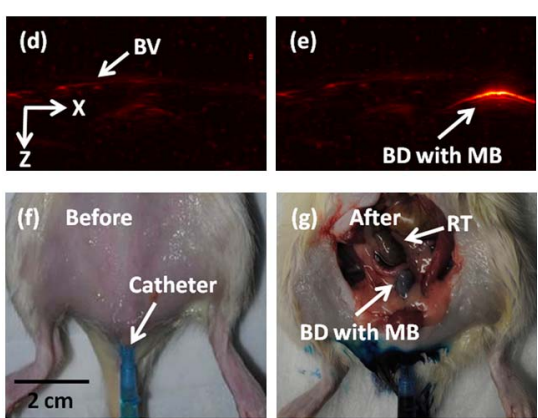

Fig. 2. (Color online) Nonionizing and noninvasive in vivo PA bladder (BD) imaging in a rat using $\mathrm{MB}$ injection. (a) Control PA image acquired at $667 \mathrm{~nm}$ in the form of MAP without MB injection. (b) PA MAP image acquired at $667 \mathrm{~nm}$ after MB injection. (c) PA MAP image acquired at $850 \mathrm{~nm}$ after MB injection. (d) PA B-scan image cut along the dotted line in (a). (e) PA B-scan image cut along the dotted line in (b). (f) Photograph with hair removed and catheterization before PA imaging. (g) Photograph with skin removed after PA imaging: BV, blood vessels; RT, rectum.

proved that MB filled the bladder. The bladder filled with $\mathrm{MB}$ is also clearly mapped in the depth-resolved PA B-scan image [Fig. 2(e)], cut along the dotted line in Fig. 2(b). The bladder was positioned $\sim 2 \mathrm{~mm}$ below the skin surface. Again, the bladder was invisible in the control PA B-scan image [Fig. 2(d)]. The accumulation of MB in the bladder was visually identified in a photograph of the same rat with the skin removed after PA imaging [Fig. 2(g)].

To explore PA mapping of deeply $(\sim 12 \mathrm{~mm})$ located bladders filled with MB, we imaged in vivo the abdominal region with chicken breast tissue placed on top of the rat. Before overlaying, a PA image was obtained to confirm the occupation of the bladder by MB. The bladder filled with MB and surrounding vasculatures are clearly seen in Fig. 3(a). With overlying chicken tissue, the bladder was successfully imaged at a depth of $12 \mathrm{~mm}$, with an image contrast of $1.13 \pm 0.25$ between the bladder and background [Fig. 3(b)]. Figures 3(c) and 3(d) show depth-resolved PA B-scan images without and with additional chicken tissue, respectively. Note that we used a laser pulse energy of less than $1 \mathrm{~mJ} / \mathrm{cm}^{2}$, which was the maximum from our laser. This value is only $1 / 20$ of the current ANSI safety limit. Kim et al. experimentally proved that they could image an MB-containing tube at a depth of $5.2 \mathrm{~cm}$ in biological tissues, using a handheld PA/US probe adapted from a clinical US imaging system and a laser pulse energy of $3 \mathrm{~mJ} / \mathrm{cm}^{2}[11]$. If the laser fluence is increased to the ANSI safety limit $\left(20 \mathrm{~mJ} / \mathrm{cm}^{2}\right)$, theoretically, the maximum penetration depth can reach $\sim 7.4 \mathrm{~cm}[\underline{11}]$. 
Without chicken tissue
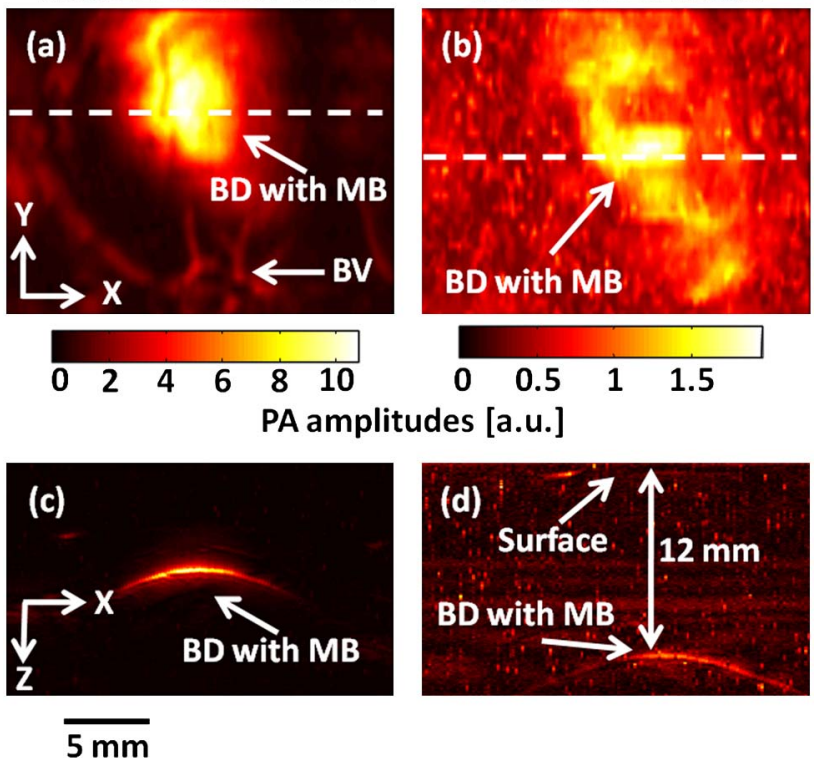

Fig. 3. (Color online) In vivo deeply penetrating PA imaging. (a) PA image acquired after MB injection and without additional chicken tissue. (b) PA image taken after MB injection with additional chicken tissue. (c) Depth-resolved PA B-scan image cut along the dotted line in (a). (d) Depth-resolved PA B-scan image cut along the dotted line in (b): BD, bladder; BV, blood vessels.

In discussion, we have shown the feasibility of a new and safe imaging method for delineating bladders. Conventional imaging techniques suffer from either ionizing radiation or low sensitivity. We have photoacoustically and spectrally imaged a rat bladder filled with clinically used MB dye in vivo. A deeply located bladder (beyond $1 \mathrm{~cm}$ ) was clearly mapped using only $1 / 20$ of the laser safety limit. Our clinical interests include monitoring vesicoureteral reflux (VUR), the retrograde flow of urine from the bladder into the upper urinary tract, in children using PAC. VUR is commonly diagnosed after patients (typically children) have a urinary tract infection [13-15]. By transporting bacteria from the bladder, VUR causes renal infections such as pyelonephritis. Therefore, early detection and treatment of VUR are essential. Two main methods have been used to evaluate VUR: fluoroscopic voiding cystourethrography (VCUG) and direct radionuclide voiding cystography (DRNC) [14]. Although radiation dose reduction has been achieved in fluoroscopic VCUG, this method is still ionizing. Further, despite the smaller radiation dose of DRNC, this technique suffers from low spatial resolution, and patients are still exposed to radiation. The pediatric VUR guidelines panel of the American Urological Association strongly urges the development of techniques with less radiation exposure [13,15]. Echo-enhanced urosonography has been applied to monitor VUR, and it offers the advantages of nonionizing, real-time, and portable imaging capability [14]. However, echoenhanced urosonography is sensitive to high-grade reflux only [16]. Therefore, US imaging alone cannot be used for VUR tracking. The nonionizing contrast-enhanced PAC can be an attractive candidate for VUR tracking and completely avoid radiation exposure to child subjects. If VUR exists, injected MB will flow to kidneys and can be photoacoustically monitored. The speckle-free nature of PAC, unlike US imaging, could further enhance the PA image contrast [17]. On the other hand, the penetration depth of PAC could be limited due to strong light scattering compared to US imaging. However, the top and bottom surfaces of a pediatric bladder are positioned at depths of $\sim 1.4$ and $\sim 4.3 \mathrm{~cm}$, respectively, from the abdominal surface, as shown in a transverse US image [18]. These depths are within the current PA imaging penetration depth, $>5 \mathrm{~cm}$ [11]. Once it is combined with a conventional US imaging probe, this technique can be portable, fast, and relatively cost effective. In the future, we will investigate PA VUR mapping in a porcine model.

We thank Drs. Saul Greenfield and Wayne Waz for fruitful scientific discussions. This work was supported by the University at Buffalo Research Foundation faculty start-up fund.

\section{References}

1. Z. Wang, C. S. D. Lee, W. C. Waltzer, J. Liu, H. Xie, Z. Yuan, and Y. Pan, J. Biomed. Opt. 12, 034009 (2007).

2. C. Nicolau, L. Bunesch, L. Peri, R. Salvador, J. M. Corral, C. Mallofre, and C. Sebastia, Brit. J. Radiol., doi:10.1259/ bjr/43400531 (2010).

3. C. Kim, C. Favazza, and L. V. Wang, Chem. Rev. 110, 2756 (2010).

4. C. Kim, E. C. Cho, J. Chen, K. H. Song, L. Au, C. Favazza, Q. Zhang, C. M. Cobley, F. Gao, Y. Xia, and L. V. Wang, ACS Nano 4, 4559 (2010).

5. C. Kim, K. H. Song, F. Gao, and L. V. Wang, Radiology 255, 442 (2010).

6. X. Wang, Y. Pang, G. Ku, X. Xie, G. Stoica, and L. V. Wang, Nat. Biotechnol. 21, 803 (2003).

7. S. Jiao, M. Jiang, J. Hu, A. Fawzi, Q. Zhou, K. K. Shung, C. A. Puliafito, and H. F. Zhang, Opt. Express 18, 3967 (2010).

8. K. Homan, J. Shah, S. Gomez, H. Gensler, A. Karpiouk, L. Brannon-Peppas, and S. Emelianov, J. Biomed. Opt. 15, 021316 (2010).

9. C. Kim, E. Todd, K. Maslov, L. Jankovic, W. J. Arkers, S. Achilefu, J. Margenthaler, M. Pashley, and L. V. Wang, J. Biomed. Opt. 15, 046010 (2010).

10. M. P. Fronheiser, S. A. Ermilov, H. P. Brecht, A. Conjusteau, R. Su, K. Mehta, and A. A. Oraevsky, J. Biomed. Opt. 15, 021305 (2010).

11. C. Kim, E. Todd, L. Jankovic, M. Pashley, and L. V. Wang, Biomed. Opt. Express 1, 278 (2010).

12. K. Song and L. V. Wang, J. Biomed. Opt. 12, 060503 (2007).

13. J. S. Elder, C. A. Peters, B. S. Arant, D. H. Ewalt, C. E. Hawrey, R. S. Hurwitz, T. S. Parrott, H. M. Snyder, R. A. Weiss, S. H. Woolf, and V. Hasselblad, J. Urol. 157, 1846 (1997).

14. K. Darge, Pediatr. Radiol. 38, 40 (2008).

15. K. L. Stratton, J. C. Pope, IV, M. C. Adams, J. W. Brock, III, and J. C. Thomas, J. Urol. 183, 2137 (2010).

16. R. L. McEwing, N. G. Anderson, S. Hellewell, and J. Mitchell, Pediatr. Radiol. 32, 853 (2002).

17. Z. Guo, L. Li, and L. V. Wang, Med. Phys. 36, 4084 (2009).

18. L. Chen, A. L. Hsiao, C. L. Moore, J. D. Dziura, and K. A. Santucci, Pediatrics 115, 1631 (2005). 